\title{
An Immune-Based Energy-Efficient Hierarchical Routing Protocol for Wireless Sensor Networks
}

\author{
Nabil Sabor ${ }^{1}, 2$, , Shigenobu Sasaki ${ }^{1}$, Mohammed Abo-Zahhad ${ }^{2}$ and Sabah M. \\ Ahmed $^{2}$ \\ ${ }^{1}$ Department of Electrical and Electronic Engineering, Niigata University, \\ Niigata 950-2181, Japan. \\ (nabil_sabor@aun.edu.eg (Correspondingauthor),shinsasaki@ieee.org) \\ ${ }^{2}$ Electrical and Electronics Engineering Department Faculty of Engineering, \\ Assiut University, Assiut 71516, Egypt. \\ (\{zahhad,sabahma\}@yahoo.com)
}

\begin{abstract}
The energy-efficiency is the primary design issue, which greatly affects the lifetime of Wireless Sensor Network (WSNs). The hierarchical-based routing is a feasible solution for reducing the energy consumption in WSNs due to reduction of the redundant data transmission. In the hierarchical routing, the network is partitioned into clusters, where each cluster consists of a head node and many member nodes. Selection of the best head nodes, that improve the lifetime and the performance of WSNs, is a NP-hard problem. Thus, this paper proposes an Immune-based Energy-Efficient hierarchical Routing Protocol (IEERP) to improve the lifetime of WSNs. IEERP utilizes the Multi-Objective Immune Algorithm (MOIA) to partition the network into optimum clusters and find locations of the best cluster heads on the basis of balancing the consumption energy among the sensor nodes and minimizing the dissipated energy in communication and overhead control packets. The operation of the proposed IEERP protocol is divided into rounds, where each round consists of two phases. The first phase is the cluster building phase, in which sink uses the MOIA algorithm to find locations of the optimum cluster heads, followed by the data transmission phase, in which the sensor nodes transfer their sensed data to the sink via the determined cluster heads. Simulation results cleared that the IEERP is more reliable protocol because it improvers the stability period and the lifetime of the homogeneous and the heterogeneous WSNs as compared to the other protocols.
\end{abstract}

Keywords: Wireless Sensor Network, Immune Algorithm, Hierarchical-based Routing, Homogeneous Networks, Heterogeneous Networks.

\section{Introduction}

Advances in digital signal processing, miniaturization of electronic circuitry, lowcost and low-power sensors have prompted the emergence of Wireless Sensor Networks (WSNs) [1]. WSNs are designed to perform a set of high-level information processing tasks such as detection, tracking and classification. The technology of WSN was developed initially for the military applications, but now it widely used in many fields such as the medical and healthcare applications, the environment and habitat applications, the industrial and civilian applications, the automation applications and the control applications [2-9]. A WSN consists of up to thousands of small size devices called sensor nodes. The sensor node is a selfpowered device with capabilities of sensing, gathering, processing, storing and wirelessly communicating. In the most cases it is very difficult and even impossible 
to change or recharge batteries of the sensor nodes due to the large number of nodes in network and the complexity of environment. Therefore, designing energy-aware routing protocols becomes an important factor for extending the lifetime of the WSNs. The routing protocols can be grouped based on the routing structure into hierarchical-based, flat-based and location-based routing protocols.

Research has proved that the hierarchical-based routing protocols perform much better than the other types in terms of the energy-efficient and the scalability. The hierarchical-based routing can effectively balance the load among the sensor nodes via assigning different task for each sensor node according to its capabilities. In the hierarchical-based routing, the network is partitioned into clusters, where each cluster contains one head node called Cluster Head $(\mathrm{CH})$ and many Member Nodes (MNs). $\mathrm{MN}$ only senses and delivers its sensed data to its related $\mathrm{CH}$, while the $\mathrm{CH}$ is responsible for collecting and aggregating the data of its MNs, then transfers the aggregated data to the Base Station (BS). Selecting the best CHs largely affects the whole network's performance and the stability. Thus, an Immune-based EnergyEfficient Hierarchical Routing Protocol (IEERP) is proposed here to improve the lifetime of the homogeneous and the heterogeneous WSNs. IEERP utilizes the Multi-Objective Immune Algorithm (MOIA) to partition the network into optimum clusters and find locations of the best $\mathrm{CHs}$ based on balancing the consumption energy among the sensor nodes and minimizing the communication cost for data and overhead control packets. MOIA algorithm is one of recently evolutionary algorithms. It has been considered here due to the following feature s [10, 11]: 1) It has the global search performance; 2) It produces the solution sets that are highly competitive in terms of convergence, diversity and distribution; 3) It has elitism, which inherently embedded in the selection mechanism to preserve good solutions and not lose them during generations; 4) It adapts the population to a reasonable size for the specific problem and this reduces the number of objective function calls; and 5) It has much less computational cost.

The rest of the paper is organized as follows. Section 2 is a literature survey about various clustering protocols. The preliminaries and formulating of the clustering problem are adopted in Section 3. Section 4 describes the proposed IEERP protocol and how the MOIA is used to find the optimum number of $\mathrm{CHs}$ and their locations. The analytical optimal number of $\mathrm{CHs}$ is proved in Section 5. In Section 6, analysis of the IEERP protocol is presented. Section 7 gives the simulation results and discussion. Finally, Section 8 offers some conclusions.

\section{Related Work}

Many hierarchical-based routing protocols for WSNs have appeared in the literature. Low Energy Adaptive Clustering Hierarchy (LEACH) protocol [12] is the first clustering protocol that has been designed especially for WSNs. LEACH partitions the network into clusters and selects $\mathrm{CHs}$ randomly. Each non- $\mathrm{CH}$ node selects the nearest $\mathrm{CH}$ to it depend on the received advertisement messages and joins to it. Once the clusters are constructed, CHs define a Time Division Multiple Access (TDMA) schedule by defining timeslots for their MNs. Each $\mathrm{CH}$ aggregates the collected data into a fixed-length packet, and forwards the aggregated packet to the BS directly. A GA-based adaptive clustering protocol, called LEACH-GA, was designed in [13] depending on the principles of LEACH protocol. The LEACH-GA protocol operates into rounds, where each round contains a setup phase and a steady-state phase. In addition, a preparation phase was added before the starting of the first round only to collect the ID and positions of all sensor nodes in the network. Based on the received information, BS begins to search for an optimal probability of CHs using GA based on minimizing the communication cost. The procedures of the setup and the steady-state phases are similar to that considered in 
the LEACH protocol. An Amend LEACH (A-LEACH) protocol was adopted in [14] for enhancing and prolonging the stability period of WSNs by considering the heterogeneity of the sensor nodes. The idea of A-LEACH protocol depends on the election probabilities of the sensor nodes in selection of CHs. The probability of each node is estimated based on its residual energy and the residual energy of the other nodes in the network. The higher-energy nodes have high probability and high chance to be $\mathrm{CH}$.

A Genetic Algorithm-based Energy-Efficient adaptive clustering hierarchy Protocol (GAEEP) [15] was suggested to efficiently prolong the stability period and the lifetime of WSNs. GAEEP prolong the lifespan of the sensor nodes in the network by determining the locations of the optimum $\mathrm{CHs}$ based on minimizing the communication cost of the sensor nodes using GA. The GAEEP protocol operates in rounds manner. Each round begins with a setup phase, in which BS constructs the optimum clusters, followed by a steady-state phase, in which $\mathrm{CHs}$ gather data of their member nodes and transfer to BS in frame formats. In [16], A Genetic Algorithm based Energy-efficient Clustering Hierarchy (GAECH) protocol was designed to extend the stability period and the lifetime of WSNs with a novel fitness function. The fitness function of GAECH considers the dissipated energy of in data collection of round, standard deviation of dissipated energy between clusters, $\mathrm{CH}$ dispersion and $\mathrm{CHs}$ energy consumption, in forming clusters. A Stable Election Protocol based on Fuzzy Logic (SEPFL) was designed in [17] to prolong the lifespan and throughput of WSNs. SEPFL utilizes the fuzzy logic control to enhance the $\mathrm{CH}$ selection and prolong the lifetime of the SEP protocol [18] depend on the distance of the sensor nodes from BS, the nodes density and the battery level of the sensor nodes. The sensor nodes with high residual energy, high probability, and requiring less energy for communicating with other nodes as well as the BS are elected as CHs. Even though the existing routing protocols can extend the lifetime of WSNs, they suffer from the load balancing and the energy hole problems. Thus, this paper proposes an Immune-based Energy-Efficient hierarchical Routing Protocol (IEERP) to extend the stability period of the homogeneous and the heterogeneous WSNs. IEERP uses the MOIA algorithm to partition the network into optimum clusters and find locations of $\mathrm{CHs}$ from the higher energy nodes based on balancing the load among the sensor nodes and minimizing the communication cost. This extends the stability period and reduces the instability period of WSNs.

\section{Preliminaries and Problem Formulation}

Let the network composes of a Base Station (BS) and N sensor nodes which are distributed in $M_{1} \times M_{2}$ target region. The network topology is represented by a connected graph $G(S, E)$, where $S=\left\{s_{l}: 0 \leq l \leq N\right\}$ is the sensor nodes set and $E$ is the set of all the possible edges formed among all the nodes in $S$. For any two nodes $s_{l}, s_{q} \in S$, there is an edge between $s_{l}$ and $s_{q}$ if and only if $\left\|s_{l}-s_{q}\right\| \leq R_{C}$, where $\left\|s_{l}-s_{q}\right\|$ is the Euclidean distance between $s_{l}$ and $s_{q}$, and $R_{C}$ is the communication range of the sensor node

\subsection{Preliminaries}

To formulate the clustering problem, the following assumptions about the sensor nodes are fixed:

- $\quad$ The BS is resource-rich device.

- $\quad$ All sensor nodes and BS are stationary after deployment.

- All sensor nodes have a unique ID and are location-aware using localization algorithms [19, 20].

- $\quad$ All sensor nodes have the ability to communicate directly with BS.

- The intra-cluster sensed information is highly correlated. 
- $\quad$ Each cluster communicates using a different CDMA code in order to reduce interference from nodes belonging to the other clusters.

- The communication channel is symmetric.

It is well known that power consumption is an important issue related to the performance of WSNs. Therefore, the first order radio model, which is shown in Figure 1, is used here for calculating the dissipated energy in transmitting and receiving k-bit packet. To transmit a k-bit message a distance $\mathrm{d}$, this radio model expends:

$$
E_{T x}(k, d)=E_{T x-\text { elec }}(k)+E_{T x-a m p}(k, d)=k E_{\text {elec }}+E_{\text {Amp }} k d^{p}
$$

and to receive this message, the radio expends:

$$
E_{R x}(k)=E_{R x-\text { elec }}(k)=k E_{\text {elec }}
$$

where, $E_{\text {elec }}$ and $E_{A m p}$ are the dissipated energy in the electronic circuit and the amplifier of the radio model respectively and $p$ is the path loss. If distance $d$ is less than the threshold distance $\left(d_{o}=\sqrt{E_{f s} / E_{m p}}\right)$, free space model $\left(f_{s}\right)$ will be used by setting $p=2$ and $E_{A m p}=E_{f s}$. Otherwise, the multipath ( $m p$ ) fading model should be adopted by setting $p=4$ and $E_{A m p}=E_{m p}$.

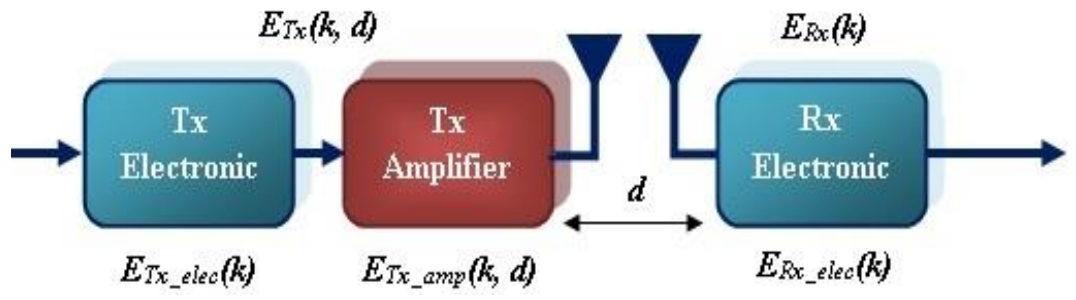

Figure 1. First Order Radio Model

Since the intra-cluster sensed information is highly correlated, $\mathrm{CHs}$ can use the data aggregation techniques to combine several correlated data signals into a single lengthfixed packet. The dissipated energy for aggregating $\mathrm{m}$ packets of $\mathrm{k}$-bits is given by:

$$
E_{a g g}(k)=m k E_{D A}
$$

Let the network is clustered into $L$ clusters and each one contains $m_{i}(i=1,2, \ldots, L)$ Member Nodes (MNs). The length of the generated data packet from each sensor node is k-bits. Each $\mathrm{MN}$ in any cluster only needs to transmit its sensed data to its respective $\mathrm{CH}$ once during a frame. Thus, the dissipated energy in the intra-cluster communication between the $\mathrm{MN} l$ and its $\mathrm{CH} i$ is given by:

$$
E_{M N-i}(l, k, d)=k E_{\text {elec }}+E_{\text {Amp }} k(d(l, i))^{p}
$$

where $d(l, i)$ is the distance between $\mathrm{MN} l$ and $\mathrm{CH} i$. On the other hand, the dissipated energy in each $\mathrm{CH}$ depends on both the intra-cluster and inter-cluster communications. Equation (6) illustrates the dissipated energy of $\mathrm{CH}$ in receiving data packets from its member nodes $\left(1^{\text {st }}\right.$ term), aggregating its own and the received packets $\left(2^{\text {nd }}\right.$ term), and transmitting the aggregated packet to BS $\left(3^{\text {rd }}\right.$ term).

$$
E_{C H}(i, k, d)=k E_{\text {elec }} m_{i}+k E_{D A}\left(m_{i}+1\right)+\left(k E_{\text {elec }}+E_{\text {Amp }} k(d(i, B S))^{p}\right)
$$

where, $d(i, B S)$ is the distance between $\mathrm{CH}$ node $i$ and BS.

\subsection{Problem Formulation}

In order to meet the performance goals of the IEERP protocol, a network must be able to adapt its behavior to accommodate changes in its intrinsic properties such as the scalability and the offered load. Percentage of CHs is important as both a large number of $\mathrm{CHs}$ and a smaller number of $\mathrm{CHs}$ would drain energy of the network. Therefore, the problem is how to partition the network into optimum $L$ clusters and find the position of their CHs $\left(P=\left\{\left(x_{1}, y_{1}\right), \ldots,\left(x_{L}, y_{L}\right)\right\}\right)$ such that the communication cost of the network is minimized, the load among the nodes is balanced, and the number of $\mathrm{CHs}$ is controlled. 
This problem can be described as a Multi-Objective Problem (MOP). The solution of this MOP will be found using the MOIA algorithm subject to the following objectives:

Objective 1: Minimize the total dissipated energy of all the sensor nodes in the network during a round in predetermined clusters as follows:

Minimize

$$
\left(E_{\text {disp }}=\sum_{i=1}^{L}\left[\left(E_{C H}(i, k, d)+E_{C H_{C P}}\left(i, k_{C P}, d\right)\right)+\sum_{l=1}^{m_{i}}\left(E_{M N-i}(l, k, d)+E_{M N_{C P}-i}\left(l, k_{C P}, d\right)\right)\right]\right)
$$

where, $E_{C H_{C P}}\left(i, k_{C P}, d\right)$ and $E_{M N_{C P}-i}\left(l, k_{C P}, d\right)$ are the dissipated energies in control packets of $\mathrm{CH} i$ and $\mathrm{MN} l$ respectively and their equations will be given in subsection 4.2.

Objective 2: Find the optimal number of $\mathrm{CHs}(L)$ for which the energy consumption will be minimal by factoring $L$ into the objective function as follows:

$$
\text { Minimize }\left(N_{C H}=L / N\right)
$$

As a result, the positions of the optimum CHs $\left(P=\left\{\left(x_{1}, y_{1}\right), \ldots,\left(x_{L}, y_{L}\right)\right\}\right)$ can be obtained by utilizing the MOIA algorithm through the minimization of the following objective function:

$$
\begin{gathered}
\text { minimize }\left(F_{I E E R P}(P)=w E_{\text {disp }}(P)+(1-w) N_{C H}(P)\right) \\
\text { subject to } E_{\text {res }}\left(C H_{i}\right) \geq E_{\text {avg }} ; i=1,2, \ldots, L
\end{gathered}
$$

where, $E_{\text {res }}\left(\mathrm{CH}_{i}\right)$ is the residual energy of $\mathrm{CH} i, E_{\text {avg }}$ is the average of the nodes' residual energy in the sensor field and $w$ is a weight factor in the range of [0 1]. The appropriate value of $w$ is based on the observed variability of each cost function.

\section{Immune-Based Energy-Efficient Hierarchical Routing Protocol}

The Immune-based Energy-Efficient Hierarchical Routing protocol (IEERP) utilizes the MOIA algorithm to solve the MOP problem by finding the optimal location of $\mathrm{CHs}$ based on minimizing the communication cost and balancing the load among the sensor nodes. The operation of the IEERP protocol is divided into rounds, where each round consists of two phases as shown in Figure 2. The first phase is the cluster building phase with duration $\mathrm{T}_{1}$. This phase is run in a hybrid manner, where the sink uses the MOIA algorithm to find locations of the optimum $\mathrm{CHs}$. Then the construction of clusters is done in a distributed mode. While, the second phase is the data transmission phase with duration $T_{2}$ which is larger than the duration of the cluster building phase (i.e. $T_{2}>T_{1}$ ) to reduce the overheads of the clustering process. The data transmission phase operates in a distributed manner, where the sensor nodes transfer their sensed data to the BS via the selected CHs. Furthermore, the information of the network is collected only one time before the first round through the Information Collection Phase (ICP) as shown in Figure 2 to reduce the control overheads. Figure 3 shows the flow chart of the IEERP protocol.

\subsection{Information Collection Phase}

The Information Collection Phase (ICP) is run only one time before the first round. In this phase, the sink initializes the network by defining the number of sensor nodes $(N)$, the initial energy of each node $\left(E_{o}\right)$, the size of data packet $(k)$, the size of sensor field $\left(\mathrm{M}_{1} \times \mathrm{M}_{2}\right)$ and parameters of the radio model. Then the sink gathers the ID, position and energy level of all sensor nodes in the network via broadcasting a Reqst_Msg(ID, $\left.x_{B S}, y_{B S}\right)$ message contains its location and ID using the flooding method. Sensor nodes that exist in the communication range of sink and receive the Reqst_Msg message, they reply by sending a Self_Info_Msg(ID, location, $\left.E_{o}\right)$ message contains its ID, location and initial energy to the sink, then they re-broadcast the request message after adding their ID in its header. A sensor node, that receives many messages from different nodes, selects the 
node that is near to it and near to the sink simultaneously and replies to it with its information. This process is repeated until the sink receives information of all sensor nodes in the network. Based on the received information from the sensor nodes, BS sets the state of all nodes to be an Ordinary Node $(\mathrm{ON})$ and stores the information in its memory. During the clustering process, any alive node in the network can be ON node or $\mathrm{MN}$ or $\mathrm{CH}$. But after building clusters, each node would be $\mathrm{CH}$ or $\mathrm{MN}$.

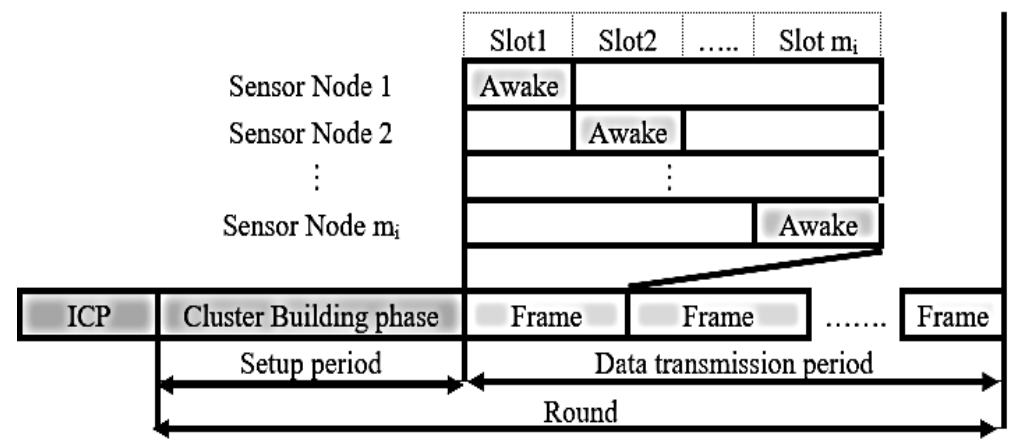

Figure 2. Operation of the IEERP Protocol

\subsection{Cluster Building Phase}

After initialization, the sink uses the MOIA algorithm to find the optimum number of $\mathrm{CHs}$ and their locations based on balancing the load among the sensor nodes and minimizing the total dissipated energy in communication process and overhead control packets of all sensor nodes as shown in Figure 3. Once CHs are determined, the sink sends a $\mathrm{CH} \_s t a t \_M s g()$ message to these node to change its state from $\mathrm{ON}$ to $\mathrm{CH}$. Based on the received messages, $\mathrm{CHs}$ advertise their state by broadcasting an $\mathrm{Adv} \_\mathrm{Msg}\left(\mathrm{CH} \_\mathrm{ID}\right)$ within their communication range. Then, each ordinary node selects the nearest $\mathrm{CH}$ to it based on the strength of the received advertising message and sends a Join_Req_Msg(MN_ID) message contains its ID to the selected $\mathrm{CH}$. CH creates a TDMA schedule by allocating timeslots according to the number of its MNs and sends a Sched_Msg(TDMA) message to inform its MNs by this schedule. The TDMA schedule is used to avoid the intra-cluster collisions and reduces the energy consumption by turning the radio of $\mathrm{MN}$ when is not in use. Also to reduce inter-cluster interference, every $\mathrm{CH}$ selects a unique CDMA code and informs all MNs within the cluster to transmit their data using this spreading code. Since the control packets consume a significant amount of energy, the IEERP protocol considers the dissipated energy of the control messages in the $\mathrm{CHs}$ selection process. The dissipated energy in the control packets for $\mathrm{CH}$ and $\mathrm{MN}$ during each round are given respectively by:

$$
\begin{aligned}
& E_{C H_{-} C P}\left(i, k_{C P}, d\right)=\underbrace{k_{C P} E_{\text {elec }}}_{\text {rec. CH_stat_Msg }}+\underbrace{k_{C P} E_{\text {elec }} m_{i}}_{\text {rec. Join_Req_Msg }}+\underbrace{2\left(k_{C P} E_{\text {elec }}+E_{\text {Amp }} k_{C P}\left(R_{C}(i)\right)^{p}\right)}_{\text {Broadcast Sched-Msg and Adv-Msg to MNs }} \\
& E_{M N_{-} C P-i}\left(l, k_{C P}, d\right)=\underbrace{2 k_{C P} E_{\text {elec }}}_{\text {rec. Adv-Msg and Sched-Msg }}+\underbrace{k_{C P} E_{\text {elec }}+E_{\text {Amp }} k_{C P}(d(l, i))^{p}}_{\text {transmit joint request to } C H i}
\end{aligned}
$$

\section{1) Selection of Cluster Heads}

Selecting the best CHs largely affects the lifetime and the stability of WSNs. Thus, The IEERP protocol utilizes the MOIA algorithm to find the optimum CHs based on balancing the consumption energy and minimizing the communication cost for data and overhead control packets. Figure 4 shows the pseudo code of the $\mathrm{CH}$ selection algorithm and its details are given below: 


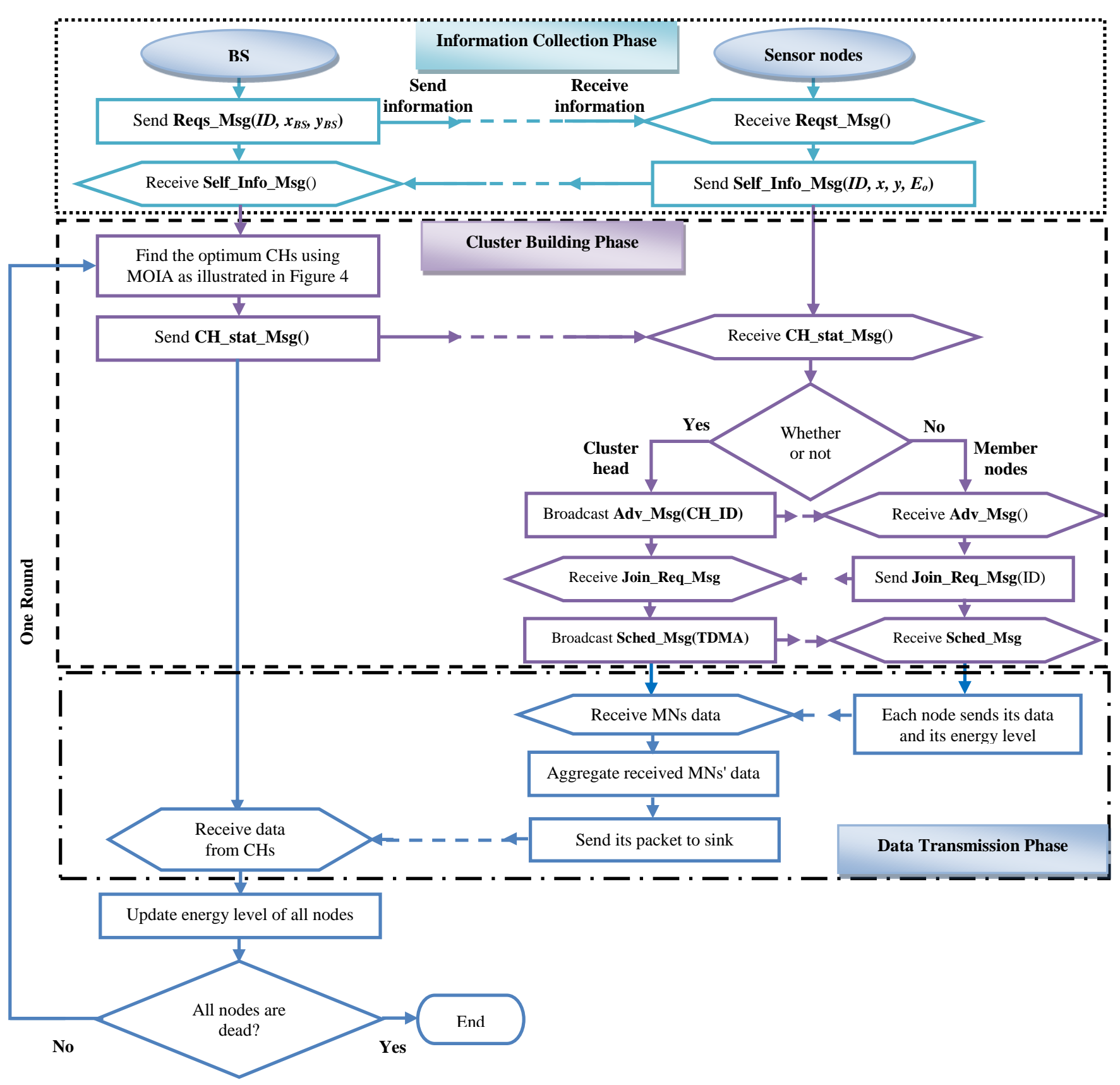

Figure 3. Flowchart of the IEERP Protocol

\section{A. Generation of Antibody Population}

Since CHs perform more tasks than the MNs, the IEERP protocol balances the consumption energy between $\mathrm{CHs}$ and $\mathrm{MNs}$ via selecting $\mathrm{CHs}$ from higher energy nodes. This can be done by constructing the candidate sensors set $\left(\mathbb{S}=\left\{\mathbb{S}_{1}, \mathbb{S}_{2}, \ldots, \mathbb{S}_{n_{\text {gen }}}\right\} ; n_{\text {gen }} \leq N\right)$ via selecting the nodes that have residual energy $\left(E_{\text {res }}\right)$ equal to or greater than the average energy $\left(E_{\text {avg }}\right)$ of all alive nodes in the sensor field as follows:

$$
\mathbb{S}=\left\{\mathbb{S}_{1}, \mathbb{S}_{2}, \ldots, \mathbb{S}_{n_{\text {gen }}}\right\}=\left\{s_{l} \mid \forall l \in\left[1 N_{\text {live }}\right] \wedge E_{\text {res }}(l) \geq E_{\text {avg }}\right\}
$$

After that, BS encodes the sensor nodes of the set $\mathbb{S}$ using a binary stream to form a population pool of $p_{s}$ antibodies, $p o p=\left(X_{1} ; X_{2} ; \ldots ; X_{m} ; \ldots ; X_{p_{s}}\right)$, as shown in Table (1). Each CHs antibody $\left(X_{m}\right)$ consists of $n_{\text {gen }}$ bits, in which each bit $\left(x_{b}\right)$ 
corresponds to one sensor in $\mathbb{S}$. A " 1 " means that the corresponding node is a $\mathrm{CH}$; otherwise, it is an ON.

Table 1. CHs Binary Antibody $\left(X_{m}\right)$

\begin{tabular}{l|c|c|c|c|c|c|c|c}
\hline \hline Sensors Set $\mathbb{S}$ & $\mathbb{S}_{\mathbf{1}}$ & $\mathbb{S}_{\mathbf{2}}$ & $\mathbb{S}_{\mathbf{3}}$ & $\mathbb{S}_{\mathbf{4}}$ & $\ldots$ & $\mathbb{S}_{\boldsymbol{b}}$ & $\ldots$ & $\mathbb{S}_{\boldsymbol{n}_{\text {gen }}}$ \\
\hline \multirow{2}{*}{ CHs Antibody $\left(\boldsymbol{X}_{\boldsymbol{m}}\right)$} & $x_{1}$ & $x_{2}$ & $x_{3}$ & $x_{4}$ & $\ldots$ & $x_{b}$ & $\ldots$ & $x_{n_{\text {gen }}}$ \\
\cline { 2 - 8 } & 1 & 0 & 1 & 1 & $\ldots$ & 0 & $\ldots$ & 1 \\
\hline \hline
\end{tabular}

Inputs: IDs, Positions, and $E_{\text {res }}$ of all sensor nodes of the set $\mathbb{S}$

Outputs: IDs and Positions of the selected CHs

$\operatorname{Set}\left(p_{s}, p_{r}, p_{c}, p_{h}, p_{m}, \operatorname{Max}_{g e n}\right)$; gen $=0$;

pop=Initial_pop $\left(p_{s}, n_{\text {gen }}\right)$;

Evaluate (pop);

While (stopping criterion is false)

gen $=$ gen +1 ;

pop_sel=RWS_Selection(pop);

pop_rep=replication ( $\left.p o p \_s e l\right)$;

pop_clon=Cloning ( $\left.p o p \_s e l\right)$;

pop_hyper=Hypermutation (pop_clon);

pop_tot=[pop_rep, pop_hyper];

pop_off=Mutation(pop_tot);

Evaluate (pop_off);

pop $=$ Const_pop(pop, pop_off);

end
$\%$ Set the parameters of MOIA

$\%$ Initialization of generations counter

$\%$ Construct an initial population with size $p_{s} \times n_{\text {gen }}$

$\%$ Evaluate the parent population

$\%$ Increment the number of generations

$\%$ Roulette wheel selection

$\%$ Selection of better antibodies

$\%$ Clonal operation

$\%$ Hypermutation operation

$\%$ Mutation operation

$\%$ Evaluate the offspring population

$\%$ Construction population of next generation

Figure 4. Pseudo Code of CHs Selection Algorithm

\section{B. Population Evaluation}

Since the most energy of the sensor nodes dissipates in the communication process [21], the IEERP protocol finds the optimum $\mathrm{CHs}$ that guarantee balancing the consumption energy among the sensor nodes and minimizing the dissipated energy in communication and overhead packets. Thus, each antibody in the population is evaluated based on the value of the objective function $F_{I E E R P}\left(X_{m}\right)$ given by Equation (8).

\section{Antibodies Selection}

In order to produce strong offspring antibodies in the next steps, a selection of better parents is performed using the Roulette Wheel Selection (RWS) mechanism [22]. The principle of RWS is based on the probability of each CHs antibody that proportions to its fitness value $\left(F i t\left(X_{m}\right)=1 / F_{\text {IEERP }}\left(X_{m}\right)\right)$. The antibodies with higher fitness values are more likely to be selected as the parent antibodies to form pop_sel $=\left(\dot{X}_{1} ; \dot{X}_{2} ; \ldots ; \dot{X}_{m} ; \ldots ; \dot{X}_{p_{s}}\right)$ which will undergo the replication and the clonal proliferation processes for generating offspring antibodies.

\section{Replication Operation}

After selecting the parent $\mathrm{CHs}$ antibodies using the RWS mechanism, these antibodies undergo the replication process. Replication process is used to select the best $p_{r} . p_{s} \mathrm{CHs}$ antibodies from pop_sel to construct pop_rep $=\left(\ddot{X}_{1} ; \ddot{X}_{2} ; \ldots ; \ddot{X}_{m} ; \ldots ; \ddot{X}_{p_{r} p_{s}}\right)$ based on the replication rate $\left(p_{r}\right)$. These antibodies will undergo the mutation process to generate offsprings. 


\section{E. Clonal Proliferation within Hypermutation}

Clonal proliferation operation is used to make the parent antibodies proliferate and produce clones. This operation is applied to the selected antibodies ( $p o p \_s e l$ ) to increase the antibodies diversity. Depending on the clonal rate $\left(p_{c}\right)$, the best $p_{s} . p_{c} \mathrm{CHs}$ antibodies are chosen from pop_sel to join the hypermutation operation to form pop_hyper $=$ $\left(\bar{X}_{1} ; \bar{X}_{2} ; \ldots ; \bar{X}_{m} ; \ldots ; \bar{X}_{p_{c} . p_{s} . N_{c l o n}}\right)$. The pop_hyper is formed via producing $N_{\text {clon }}$ clones for each antibody through mutating some bits in each antibody based on hypermutation rate $\left(p_{h}\right)$. If the antibody $\dot{X}_{m}=\left\{x_{1}, x_{2}, \ldots, x_{a}, \ldots, x_{b}, \ldots, x_{c}, \ldots, x_{n_{g e n}}\right\}$ is chosen depending on $p_{c}$, the resulting clone is $\bar{X}_{m}=\left\{x_{1}, x_{2}, \ldots, x_{a}^{\prime}, \ldots, x_{b}^{\prime}, \ldots, x_{c}^{\prime}, \ldots, x_{n_{g e n}}\right\}$, where $x_{a, b, c}^{\prime}=1-$ $x_{a, b, c}$. Table (2) shows the effect of hypermutation on the single antibody $\left(\dot{X}_{m}\right)$ that is selected based on $p_{c}$ to produce two clones. The shaded bits in Table (2) are the bits that mutated from 0 to 1 or vice versa based on $p_{h}$.

Table 2. An Example of Hypermutation

\begin{tabular}{c|c|c|c|c|c|c|c|c}
\hline \hline Original Antibody $\left(\dot{X}_{m}\right)$ & 1 & 0 & 1 & 1 & $\ldots$ & 0 & $\ldots$ & 1 \\
\hline First clone Antibody $\left(\bar{X}_{m 1}\right)$ & 0 & 1 & 1 & 0 & $\ldots$ & 1 & $\ldots$ & 1 \\
\hline Second clone Antibody $\left(\bar{X}_{m 2}\right)$ & 1 & 1 & 0 & 1 & $\ldots$ & 0 & $\ldots$ & 1 \\
\hline \hline
\end{tabular}

\section{F. Mutation Operation}

Mutation alters one or more genes depending on the mutation rate $p_{m}$ to provide exploration. The mutation operation is used to mutate the replicated population ( $p o p_{-} r e p$ ) and hypermutated population (pop_hyper) to produce offspring population pop_off $=$ $\left(\overline{\bar{X}}_{1} ; \overline{\bar{X}}_{2} ; \ldots ; \overline{\bar{X}}_{m} ; \ldots ; \overline{\bar{X}}_{l_{o f f}}\right)$, where $l_{o f f}=p_{s} . p_{c} . N_{c l o n}+p_{s} . p_{r}$. For a given antibody $\bar{X}_{m}=$ $\left\{x_{1}, x_{2}, \ldots, x_{b}, \ldots, x_{n_{g e n}}\right\}$, if the bit $x_{b}$ is selected for mutation depending on $p_{m}$, the resulting offspring is $\bar{X}_{m}=\left\{x_{1}, x_{2}, \ldots, x_{b}^{\prime}, \ldots, x_{n_{\text {gen }}}\right\}$. The new bit $x_{b}^{\prime}$ is a complement of $x_{b}$. In our protocol, the mutation rate is set by $p_{m}=1 / n_{\text {gen }}$ as stated in [23], where $n_{\text {gen }}$ is the length of CHs antibody $\left(\bar{X}_{m}\right)$. Table (3) shows the effect of mutation on the hypermutated antibody $\left(\bar{X}_{m}\right)$. Based on $p_{m}$, the shaded bits are chosen to mutate from 0 to 1 or vice versa.

\section{Table 3. An Example of Mutation}

\begin{tabular}{c|c|c|c|c|c|c|c|c}
\hline \hline Hypermutated Antibody $\left(\overline{\boldsymbol{X}}_{\boldsymbol{m} 1}\right)$ & 0 & 1 & 1 & 0 & $\ldots$ & 1 & $\ldots$ & 1 \\
\hline Mutated Antibody $\left(\overline{\bar{X}}_{\boldsymbol{m}}\right)$ & 1 & 1 & 1 & 0 & $\ldots$ & 0 & $\ldots$ & 1 \\
\hline \hline
\end{tabular}

\section{G. Population Updating}

A population is updated as a result of completing one generation. Based on $F_{\text {IEERP }}$ values, $p_{s}$ antibodies are selected from the initial population $\left(\right.$ pop $\left.=\left(X_{1} ; X_{2} ; \ldots, X_{m} ; \ldots ; X_{p_{s}}\right)\right) \quad$ and offspring population $\left(\right.$ pop_off $\left.=\left(\overline{\bar{X}}_{1} ; \overline{\bar{X}}_{2} ; \ldots ; \overline{\bar{X}}_{n} ; \ldots ; \overline{\bar{X}}_{l_{o f f}}\right)\right)$ to form the population of the next generation $\left(\right.$ pop $\left.=\left(X_{1} ; X_{2} ; \ldots, X_{m} ; \ldots ; X_{p_{s}}\right)\right)$; where $X_{m}$ is given by:

$$
X_{m}=\left\{\begin{array}{l}
\overline{\bar{X}}_{n}, \forall n \in\left[\begin{array}{ll}
1 & l_{\text {off }}
\end{array}\right], \text { if } F\left(\overline{\bar{X}}_{n}\right)<F\left(X_{m}\right) \\
X_{m}, m=1,2, \ldots, p_{s}
\end{array}\right.
$$




\section{H. Stopping Criterion}

The MOIA algorithm converges to the optimum $\mathrm{CHs}$ when $F_{\text {IEERP }}$ of the population does not change for a certain number of generations or when the number of generations exceeds the maximum number of generations $\left(\operatorname{Max}_{\mathrm{gen}}\right)$.

\section{2) C Clusters Construction}

Once the CHs are found, BS sends a $\mathrm{CH}_{-}$stat_Msg() message to change the state of the sensor nodes from "ON" to "CH". Based on the received message, the node changes its state to $\mathrm{CH}$ and broadcasts an $A d v_{-} M s g\left(C H \_I D\right)$ message within its $R_{C}$ to advertise its state to the ordinary nodes in the network. Depending on the received advertising messages from $\mathrm{CHs}$, each ordinary node sends a Join_Req_Msg(MN_ID) message contains its ID to the nearest $\mathrm{CH}$ that covers it based on the strength of the received messages. Then, each $\mathrm{CH}$ creates the TDMA schedule by allocating timeslots according to the number of its MNs and broadcasts a Sched_Msg(TDMA) message within its $R_{C}$ to inform its MNs by this schedule. The TDMA schedule is used to avoid intra-cluster collisions and allows for each member node to turn its radio off, except during its transmission time, to minimize the energy consumption.

\subsection{Data Transmission Phase}

Once the clusters are constructed, the MNs wake up and sense data. Then, each node sends the sensed data and its residual energy to its corresponding $\mathrm{CH}$ during its determined timeslot. This method allows sensor nodes to sleep during other timeslots to save their energy. It should be noted that each $\mathrm{CH}$ node must keep its receiver on to receive data from all nodes in the cluster. Each cluster communicates using a different CDMA code in order to reduce the interference of nodes belonging to the other clusters. Once each $\mathrm{CH}$ receives the sensed data from its $\mathrm{MNs}$, it performs signal processing functions to aggregate the data into a single packet. Then, $\mathrm{CHs}$ send the resulting packets and residual energy of their MNs to BS. After a certain time, which is determined a priori, the network begin a new round and runs the cluster building phase to select new $\mathrm{CHs}$ using the MOIA algorithm.

\section{Analytical Optimal Number of CHs}

In this section, we can analytically determine the optimal number of $\mathrm{CHs}$ based on minimizing the total dissipated energy of all the sensor nodes in the $M_{1} \times M_{2}$ field. From Equations (4)-(5) and (9)-(10), the total dissipated energy of all sensor nodes is given by:

$$
E_{\text {disp }}=\left(\sum_{i=1}^{L}\left[\left(E_{C H}(i, k, d)+E_{C H_{C P}}\left(i, k_{C P}, d\right)\right)+\sum_{l=1}^{m_{i}}\left(E_{M N-i}(l, k, d)+E_{M N_{C P}-i}\left(l, k_{C P}, d\right)\right)\right]\right)
$$

If each cluster contains the same number of MNs (i.e. $m_{i}=\frac{N}{L}-1 \approx \frac{N}{L}$ ), then the total dissipated energy can be simplified to:

$$
E_{\text {disp }} \approx L\left(2 \frac{N}{L} k E_{\text {elec }}+\frac{N}{L} k E_{D A}+k E_{\text {Amp }} d_{\text {toBS }}^{p}+4 \frac{N}{L} k_{C P} E_{\text {elec }}+\frac{N}{L}\left(k+k_{C P}\right) E_{f S} E\left[d_{\text {toCH }}^{2}\right]\right)
$$

Where $E\left[d_{t o C H}^{2}\right]$ is the expected square distance from the node to $\mathrm{CH}$. The area occupied by each cluster is approximately $\left(M_{1} \times M_{2}\right) / L$. In general, this is an arbitrary-shaped region with a node distribution $\rho(x, y)=1 /\left(\left(M_{1} \times M_{2}\right) / L\right)$. Since the area of each cluster is a circle with radius $R_{c}=\sqrt{\left(M_{1} \times M_{2}\right) / \pi L}$, the expected squared distance from the nodes to the cluster head (assumed to be at the center of the cluster) is given by:

$$
\begin{aligned}
E\left[d_{t o C H}^{2}\right] & =\iint\left(x^{2}+y^{2}\right) \rho(x, y) d x d y=\iint_{M_{1} \times M_{2}} r^{2} \rho(x, y) r d r d \theta \\
& =\left(\frac{L}{M_{1} \times M_{2}}\right) \int_{\theta=0}^{2 \pi} \int_{r=0}^{R_{c}} r^{3} d r d \theta=\frac{1}{2 \pi L}
\end{aligned}
$$

From Equation (14) and (15), we have

$$
E_{\text {disp }} \approx 2 N k E_{\text {elec }}+N k E_{D A}+L k E_{A m p} d_{\text {toBS }}^{p}+4 N k_{C P} E_{\text {elec }}+N\left(k+k_{C P}\right) E_{f S} \frac{M_{1} \times M_{2}}{2 \pi L}(16)
$$


By differentiating Equation (16) with respect to $\mathrm{L}$ and setting it to zeros, the optimal number of clusters (Lopt) can be found:

$$
L_{\text {opt }}=\sqrt{\frac{N}{2 \pi}} \sqrt{\frac{k+k_{C P}}{k}} \sqrt{\frac{E_{f s}}{E_{A m p}}} \sqrt{\frac{M_{1} \times M_{2}}{d_{t o B S}^{p}}}
$$

If distance $d_{t o B S}$ is less than the threshold distance $\left(d_{o}=\sqrt{E_{f s} / E_{m p}}\right)$, the free space model will be used by setting $p=2$ and $E_{\text {Amp }}=E_{f s}$. Otherwise, the multipath fading model should be adopted by setting $p=4$ and $E_{A m p}=E_{m p}$.

\section{Analysis Of IEERP Protocol}

Lemma 1: The overhead complexity of control messages for the IEERP clustering protocol in the network is $\mathrm{O}(N)$ and the time complexity is $\mathrm{O}\left(n_{\text {gen }}\right)$.

Proof: Since the information collection phase exists only in the first round and its duration is very small, we neglect the overhead packets in it. As the cluster building phase is run in each round, it adds overheads to the protocol. In this phase, each member node sends a Join_Req_Msg() message to its respective $\mathrm{CH}$. Also each $\mathrm{CH}$ node receives a CH_stat_Msg() from BS and broadcasts an $A d v \_M s g()$ and a Sched_Msg() messages to its MNs. If the number of CHs is $L$, the total number of $C H \_s t a t \_M s g()$ or Sched_Msg() or $A d v_{-} M s g()$ messages is equal $L$ and the number of Join_Req_Msg() messages is $(N-L)$. Thus, the total number of the overhead control messages for clustering in each round adds up to:

$$
L+L+L+(N-L)=N+2 L
$$

So that the overhead complexity of the control messages for the IEERP is $\mathrm{O}(N)$. IEERP adopts a centralized clustering strategy to find the optimum CHs from only $n_{\text {gen }}\left(n_{\text {gen }} \leq N\right)$ sensor nodes that have residual energy $\left(E_{r e s}\right)$ equal to or greater than the average energy $\left(E_{\text {avg }}\right)$ of all alive nodes in the sensor field. The worst case occurs in the first roud when all sensor nodes have the same initial energy. Thus, the time complexity of the entire network is $O\left(n_{\text {gen }}\right)$.

Lemma 2: The computational complexity of the IEERP protocol is $O\left(\operatorname{Max}_{\text {gen }} \cdot n_{\text {gen }} \cdot N_{\text {clon }} \cdot p_{s}\right)$.

Proof: Since the IEERP protocol utilizes the MOIA algorithm in finding the optimum clusters, the complexity of the IEERP protocol depends on the complexity of MOIA algorithm. The MOIA algorithm consists of five major operations: the antibodies selection, the replication, the clonal proliferation with hypermutation, the mutation and the population updating. Assume that the population consists of $p_{s}$ antibodies and the length of each antibody is $n_{g e n}$ genes. The selection operation requires $p_{s}$ calculation times to the selected $p_{s}$ antibodies and the number of calculations in the replication operation does not exceed $p_{r} . p_{s}$. The clonal operation selects $p_{c} \cdot p_{s}$ antibodies to produce $N_{c l o n}$ for each one via hypermutation operation, so the total number of clones $\left(N_{\text {clon }} \cdot p_{c} \cdot p_{s}\right)$ requires $p_{h} \cdot n_{g e n} \cdot N_{\text {clon }} \cdot p_{c} \cdot p_{s}$ calculations. The mutation operation needs $p_{m} \cdot n_{g e n} \cdot\left(p_{r} \cdot p_{s}+N_{c l o n} \cdot p_{c} \cdot p_{s}\right)$ calculations to mutate the replicated antibodies and the hypermutated offspring antibodies. The final step is the population updating which requires $p_{s}$ calculations to select $p_{s}$ antibodies of the new population. In generation gen, the total number of $C$ (gen) calculations meets:

$C($ gen $)=p_{s}+p_{r} \cdot p_{s}+p_{h} \cdot n_{\text {gen }} \cdot N_{\text {clon }} \cdot p_{c} \cdot p_{s}+p_{s}+p_{m} \cdot n_{g e n} \cdot\left(p_{r} \cdot p_{s}+N_{\text {clon }} \cdot p_{c} \cdot p_{s}\right)(19)$ Therefore, if the total number of generations is $\operatorname{Max}_{\text {gen }}$, the computational complexity of the IEERP is approximate to $O\left(\operatorname{Max}_{\text {gen }} . n_{\text {gen. }} . N_{c l o n} . p_{s}\right)$. When $p_{r}, p_{c}, p_{h}$ and $p_{m}$ are set to 1 , $n_{\text {gen }}$ equals $N$ and each antibody in the population produces $p_{s}$ clones (i.e. $N_{c l o n}=p_{s}$ ), the worst case running time is considered. In this case, $C($ gen $)$ will be given by (20) which shows that the worst IEERP complexity is $O\left(\operatorname{Max}_{g e n} \cdot N \cdot p_{s}{ }^{2}\right)$.

$$
C(\text { gen }) \leq p_{s}+p_{s}+N \cdot p_{s}{ }^{2}+N \cdot\left(p_{s}+p_{s}{ }^{2}\right)+p_{s}=(N+3) p_{s}+2 N \cdot p_{s}{ }^{2}
$$




\section{Simulations and Results}

In this section, we perform a series of simulation scenarios using Matlab (R2013a) to investigate the feasibility and effectiveness of the IEERP protocol compared to other protocols.

\subsection{Performance Metrics}

These metrics are used to evaluate the performance of the IEERP protocol [24, 25]:

- Network Lifetime: The time interval from the start of network operation until the death of the last alive sensor node.

- Stability Period: The time interval from the start of network operation until the death of the first sensor node.

- Instability Period: The time interval from the death of the first sensor node until the death of the last sensor node.

- $\quad$ First Dead Node (FDN): The number of rounds after which the first sensor node is died.

- $\quad$ Last Dead Node (LDN): The number of rounds after which all sensor nodes are died.

- $\quad$ Number of Alive Nodes per Round: The number of nodes that have not yet expended all of their energies.

- Balanced Extent of Energy Dissipation (BEED): It measures the ability of the protocol to balance the dissipated energy and is calculated as BEED $=(\mathrm{LDN}-$ FDN)/LDN. The protocol with smaller BEED has a better performance in aspect of balancing energy dissipation.

\subsection{Simulations and Discussion}

In the conducted simulations, we assumed that the sensor nodes sense the environment continuously and send the data at a fixed rate. Each simulation was run for 10 different networks and the average was taken as the final result to eliminate the experimental error caused by randomness. For fair comparison with other protocols, the energy consumption due to communication is calculated using the first-order energy model with these parameters $E_{\text {elec }}=50 \mathrm{~nJ} / \mathrm{bit}, E_{f s}=10 \mathrm{pJ} / \mathrm{bit} / \mathrm{m}^{2}, E_{m p}=0.0013 \mathrm{pJ} / \mathrm{bit} / \mathrm{m}^{4}$ and $E_{D A}=5$ $n J /$ bit/signal. The MOIA parameters are set based on the study presented in [23] as $p_{s}=30$, $p_{r}=0.9, p_{c}=0.05, p_{h}=10 p_{m}, N_{c l o n}=5$ and $\mathrm{Max}_{\mathrm{gen}}=150$. The weight factor of the objective function is set as $w=0.9$.

\section{1) Performance of IEERP}

In this simulation, we investigate the performance of IEERP protocol compared to LEACH [12] and GAECH [16] protocols. The simulation consists of 100 homogeneous nodes with initial energy of $1 \mathrm{~J}$, scattered randomly within a $100 \times 100 \mathrm{~m}^{2}$ sensor field. The packets sent were 4000 bits plus 200 bits control packets. The BS is located at the center of the sensor field $(50 \mathrm{~m}, 50 \mathrm{~m})$. The obtained clustered network using the IEERP protocol is shown in Figure 5. Figure 6 shows the obtained lifetime for the IEERP, LEACH and GAECH protocols. The average energy consumption per round, BEED, FDN and LDN metrics for the three protocols are given in Table (4). From the obtained results, it is noticed that the IEERP protocol outperforms the other protocols in terms of balancing the energy consumption, stability period and network lifetime. Since the IEERP protocol selects $\mathrm{CHs}$ from the higher energy nodes, it balances the energy consumption among the sensor nodes which reduces the instability period as cleared from BEED values. Also, it improves the stability period by $19.29 \%$ and $4 \%$ compared to LEACH and GAECH respectively and the overall lifetime by $17.02 \%$ and $3.25 \%$. Since the BS is located at center of the field, the distances from CHs to BS $\left(d_{t o B S}\right)$ vary from $30 \mathrm{~m}$ to 70 m. Therefore, the analytical optimal number of $\mathrm{CHs}\left(L_{\text {opt }}\right)$ according to Equation (17) is 
varied from 6 to $14 \mathrm{CH}$. Figure 7 shows the obtained number of $\mathrm{CHs}$ using the IEERP protocol against the number of rounds. It is observed that the obtained number of CHs using the IEERP is approximately uniform along all rounds and varies from 7 to $9 \mathrm{CH}$ which lies in the analytical range.

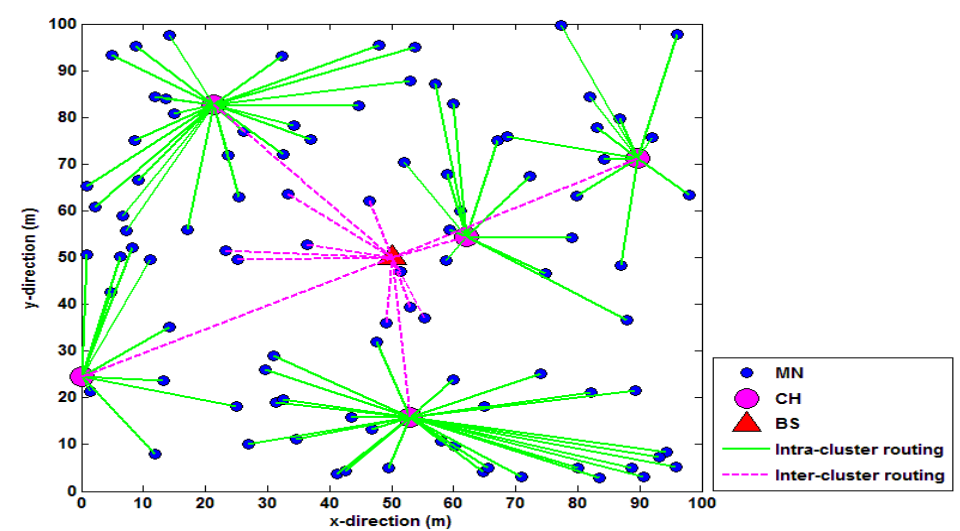

Figure 5. The Clustered Network of Simulation 1

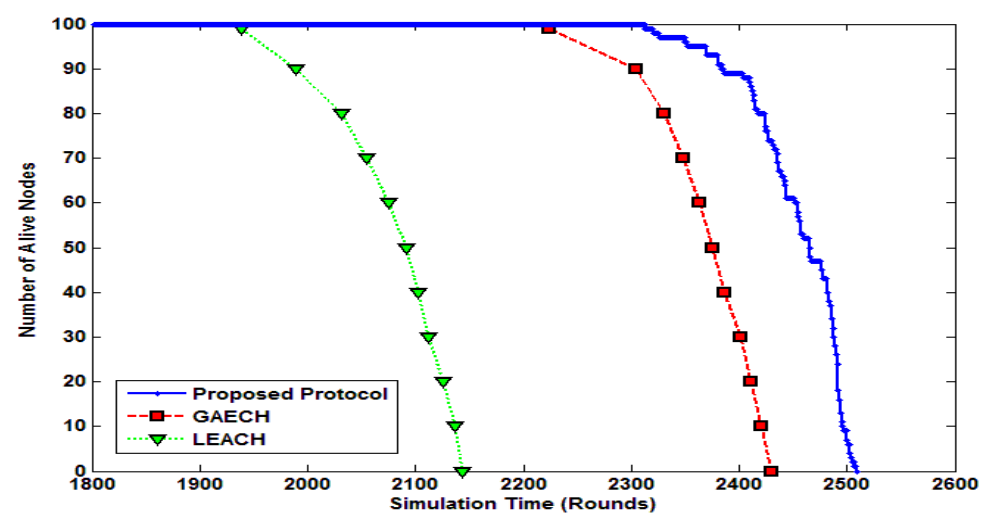

Figure 6. Network Lifetime of Simulation 1

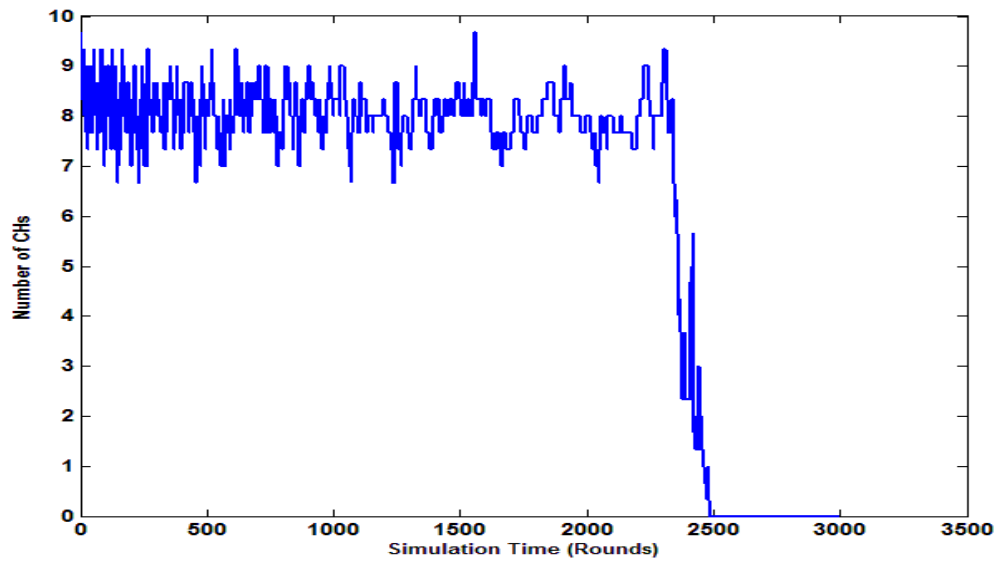

Figure 7. Obtained Number of CHs using the IEERP Protocol of Simulation 1 
Table 4. Obtained Results of Simulation 1

\begin{tabular}{c|c|c|c|c}
\hline \hline Protocol & $\begin{array}{c}\text { Average energy consumption } \\
\text { per round }(\mathbf{J})\end{array}$ & BEED & FDN & LDN \\
\hline LEACH [12] & 0.046 & 0.0953 & 1939 & 2144 \\
\hline GAECH [16]. & 0.042 & 0.085 & 2224 & 2430 \\
\hline IEERP & $\mathbf{0 . 0 4 0 4}$ & $\mathbf{0 . 0 7 8 1}$ & $\mathbf{2 3 1 3}$ & $\mathbf{2 5 0 9}$ \\
\hline \hline
\end{tabular}

\section{2) Effect of BS location}

Since the location of BS is having a considerable effect on the energy consumption, we study the effect of BS position on the performance of the proposed IEERP protocol and compare its performance with LEACH [12], LEACH-GA [13], A-LEACH [14] and GAEEP [15] protocols. The simulation consists of 100 homogeneous nodes with initial energy of $0.5 \mathrm{~J}$, scattered randomly within a $50 \times 50 \mathrm{~m}^{2}$ sensor field. The packets sent were 2000 bits plus 50 bits control packets. Here, we consider three cases for the location of BS; namely are 1) BS is located at center of the field $(25 \mathrm{~m}, 25 \mathrm{~m}) ; 2)$ BS is located on the edge of the field ( $25 \mathrm{~m}, 50 \mathrm{~m})$; and 3) BS is located out the field (25 m, $100 \mathrm{~m})$. Table (5) and Figs. (8-10) show FDN, HDN and LDN metrics respectively of LEACH, LEACHGA, A-LEACH, GAEEP and IEERP protocols for three cases of BS.

From the obtained results, it is noticed that the number of rounds is large when the BS is located at the center of sensor field and it decreases when the BS moves outward. Also, it is obvious that the stability period of the IEERP protocol extended as compared to LEACH, LEACH-GA, A-LEACH and GAEEP by 1033, 890, 880 and 290 rounds respectively when BS at $(25 \mathrm{~m}, 25 \mathrm{~m})$ and by $824,814,658$, and 240 rounds respectively when BS at $(25 \mathrm{~m}, 100 \mathrm{~m})$. It is observed that the stability period obtained using the IEERP protocol when the BS at center of the sensor field is wider than that obtained when BS moves outward. This due to using the free space communication when the BS located at $(25 \mathrm{~m}, 25 \mathrm{~m})$ because the distances between BS and the sensor nodes always less than the threshold distance $\left(d_{o}\right)$. This decreases the dissipated energy and extends the stability period. However, the distances between BS and the sensor nodes increase when the BS moves outward and this increases the dissipated energy due to using the multipath communication. Moreover, the instability period is narrow as compared the other protocols and this increases the reliability of the IEERP protocol in the clustering process of WSN. From results of LDN metric, it is cleared that the IEERP protocol improves the network lifetime by $43.78,30.39,4.89$ and $2.31 \%$ as compared to LEACH, LEACH-GA, A-LEACH and GAEEP respectively when BS is located at center of the sensor field. When BS is located at $(25 \mathrm{~m}, 100 \mathrm{~m})$, the network lifetime improved by 36.61, 23.19, 4.40 , and $2.81 \%$ compared the other protocols. The network lifetime when BS is located outside the field is improved by $31.51,29.48,6.38,1.29 \%$ compared to the other protocols. 


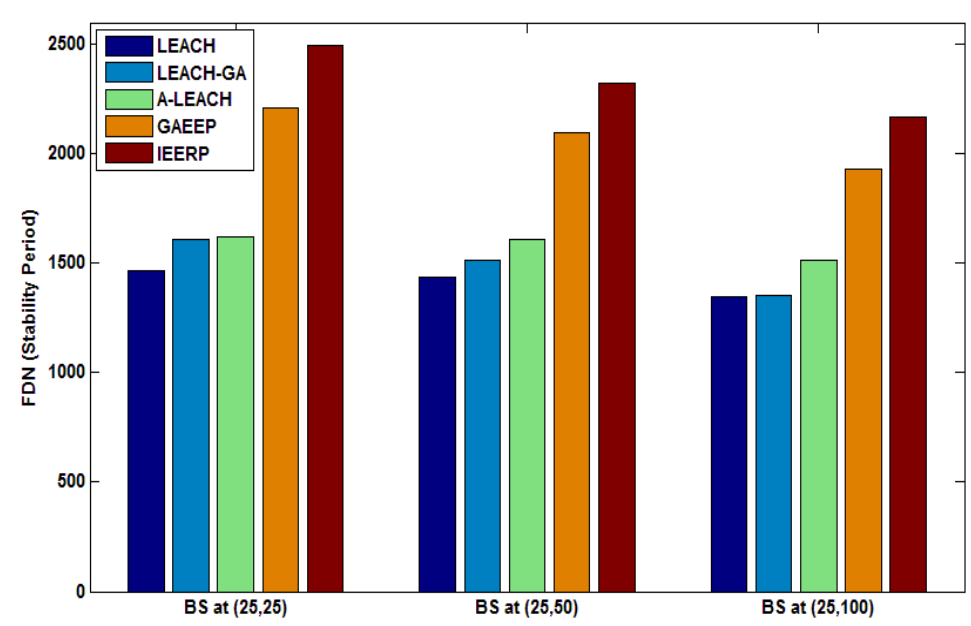

Figure 8. FDN obtained at different Location of BS

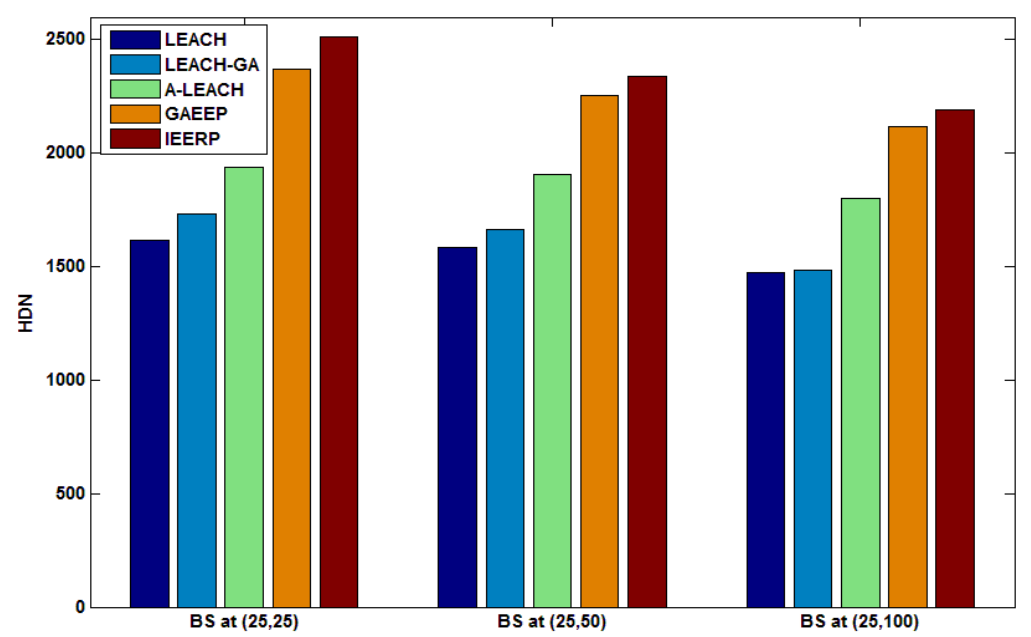

Figure 9. HDN obtained at different Location of BS

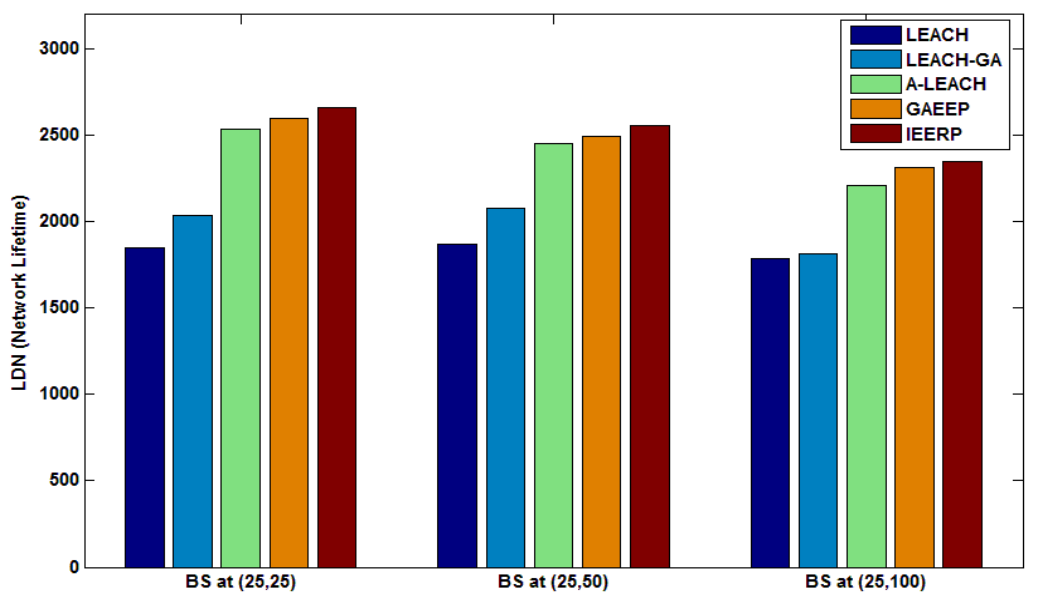

Figure 10. LDN Obtained at different Location of BS 
Table 5. Obtained Results of Simulation 2

\begin{tabular}{c|c|c|c|c|c|c}
\hline $\begin{array}{c}\text { BS } \\
\text { Location } \\
(\mathbf{m}, \mathbf{m})\end{array}$ & Metric & $\begin{array}{c}\text { LEACH } \\
{[12]}\end{array}$ & $\begin{array}{c}\text { LEACH-GA } \\
{[13]}\end{array}$ & $\begin{array}{c}\text { A-LEACH } \\
{[14]}\end{array}$ & $\begin{array}{c}\text { GAEEP } \\
{[15]}\end{array}$ & IEERP \\
\hline \multirow{3}{*}{$(\mathbf{2 5}, \mathbf{2 5})$} & FDN & 1467 & 1610 & 1620 & 2210 & $\mathbf{2 5 0 0}$ \\
\cline { 2 - 7 } & HDN & 1691 & 1818 & 2209 & 2370 & $\mathbf{2 5 1 0}$ \\
\cline { 2 - 7 } & LDN & 1850 & 2040 & 2536 & 2600 & $\mathbf{2 6 6 0}$ \\
\hline & FDN & 1438 & 1512 & 1608 & 2100 & $\mathbf{2 3 2 0}$ \\
\cline { 2 - 7 }$(\mathbf{2 5}, \mathbf{5 0})$ & HDN & 1661 & 1717 & 2186 & 2260 & $\mathbf{2 3 4 0}$ \\
\cline { 2 - 7 } & LDN & 1874 & 2078 & 2452 & 2490 & $\mathbf{2 5 6 0}$ \\
\hline & FDN & 1346 & 1356 & 1512 & 1930 & $\mathbf{2 1 7 0}$ \\
\cline { 2 - 7 }$(\mathbf{2 5}, \mathbf{1 0 0})$ & HDN & 1543 & 1554 & 2077 & 2120 & $\mathbf{2 1 9 0}$ \\
\cline { 2 - 7 } & LDN & 1787 & 1815 & 2209 & 2320 & $\mathbf{2 3 5 0}$ \\
\hline \hline
\end{tabular}

\section{3) Effect of Nodes Heterogeneity}

The effect of the node heterogeneity on the performance of the IEERP protocol is studied in this simulation compared to the SEP [18] and the SEPFL [17] protocols. Simulation consists of 100 heterogeneous sensor nodes that are randomly distributed within a $100 \times 100$ field. $20 \%$ of the total nodes are advanced nodes having $100 \%$ more energy than the normal ones such that the total residual energy of all nodes are $100 \mathrm{~J}$. The BS is located at the center of the field and the packets sent were 4000 bits. Figs. (11-13) shows the network lifetime, the energy dissipation rate and the packet throughput for the three protocols. The FDN, HDN, LDN and BEED matrices are listed in Table (6). It is seen that the IEERP protocol prolongs the stability period by 1112 and 925 rounds and the overall lifetime by 1702 and 0 rounds as compared to the SEP and the SEPFL protocols respectively. Moreover, it balances the energy consumption among the sensor nodes via selecting the higher energy nodes to be $\mathrm{CHs}$ as cleared from the values of BEED. The dissipation energy rate of the IEERP protocol is smother than the other protocols until to the round 2200 which extends the stability period. After that, its dissipation rate downs below the rate of the SEPFL protocol due to balancing the load among the sensor nodes. Extending the stability period using the IEERP protocol allows the BS to gathers more packets as shown in Figure 13. Thus, the IEERP improves the network throughput by 72.28 and $128.76 \%$ as compared to the other two algorithms.

Table 6. FDN, HDN, LDN and BEED Metrics of Simulation 3

\begin{tabular}{c|c|c|c|c}
\hline Protocol & FDN & HDN & LDN & BEED \\
\hline SEP [18] & 1007 & 1500 & 2325 & 0.5669 \\
\hline SEPFL [17] & 1194 & 2092 & 4027 & 0.7035 \\
\hline IEERP & $\mathbf{2 1 1 9}$ & $\mathbf{2 4 9 7}$ & $\mathbf{4 0 2 7}$ & $\mathbf{0 . 4 7 3 8}$ \\
\hline
\end{tabular}




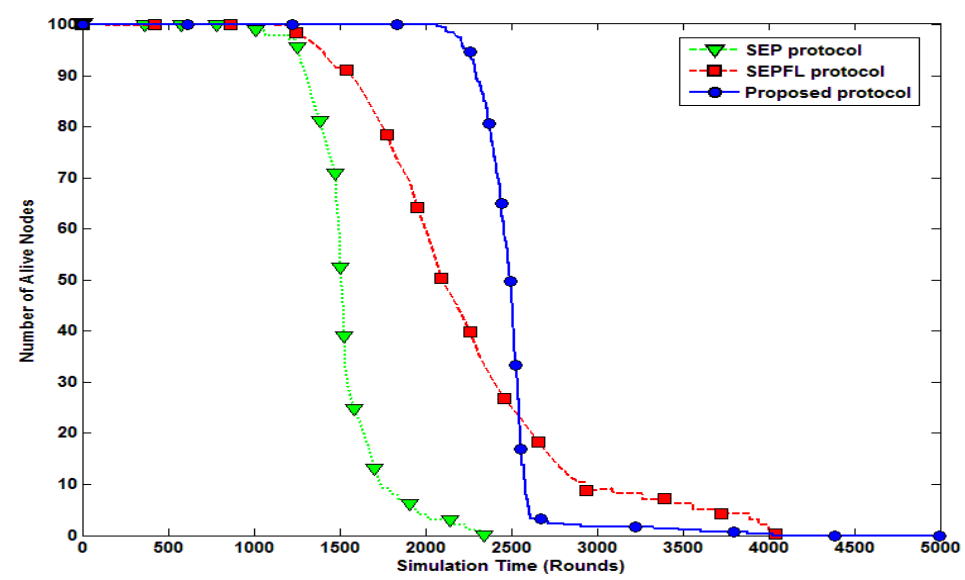

Figure 11. Network Lifetime of Heterogeneous Network (Simulation 3)

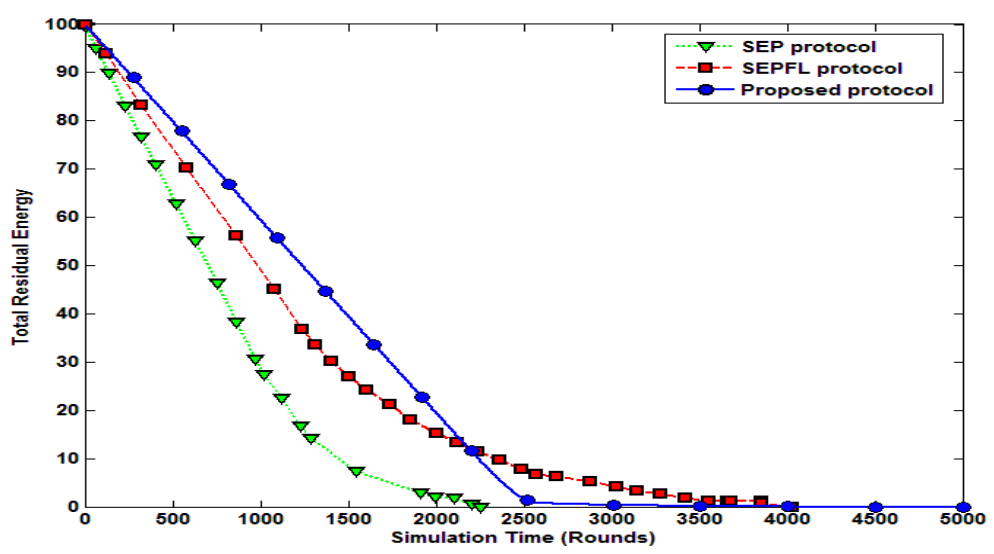

Figure 12. Energy Dissipation Rate of Heterogeneous Network (Simulation 3)

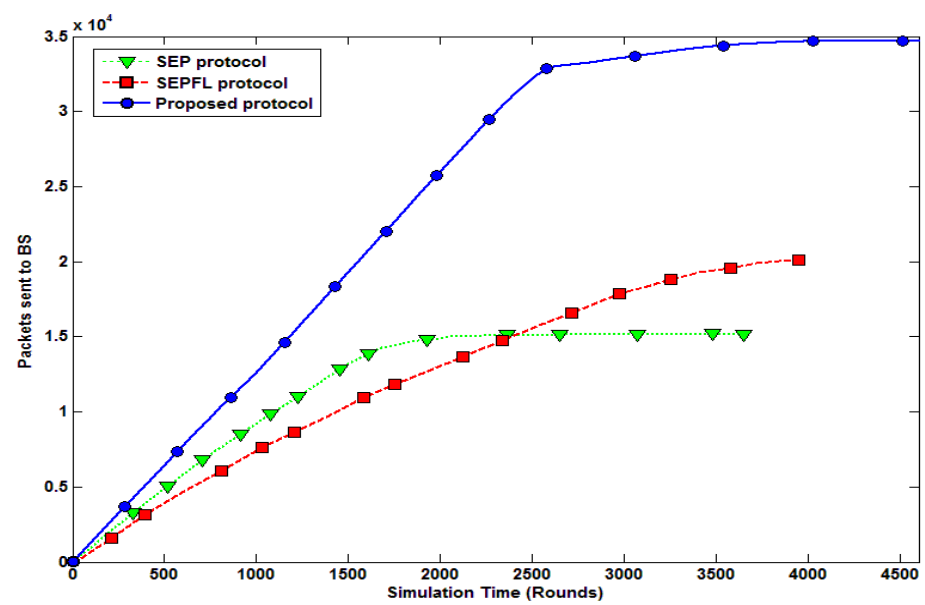

Figure 13. Network Throughput of Heterogeneous Network (Simulation 3)

\section{Conclusion}

In this paper, an Immune-based Energy-Efficient hierarchical Routing Protocol (IEERP) has been presented to efficiently maximize the lifetime and the stability period of the homogeneous and the heterogeneous wireless sensor networks. The operation of the IEERP protocol is divided into rounds, where each round consists of a cluster building 
phase and a data transmission phase. In the cluster building phase, IEERP utilizes the Multi-Objective Immune Algorithm to partition the network into optimum clusters and find locations of the best cluster heads on the basis of balancing the consumption energy among the sensor nodes and minimizing the communication cost for data and overhead control packets. While in the data transmission phase, each cluster head gathers and aggregates data of its member node and then forwards the aggregated data to the base station. Analytical and simulation results showed that the proposed IEERPP protocol is more energy-efficient and more reliable because it expands the stability period and compresses the instability period. Moreover, it outperforms the previous protocols in terms of the energy dissipation rate, the network lifetime, the stability period and the load balancing.

\section{Acknowledgments}

The authors would like to acknowledge the cultural affairs and mission sector of Egyptian Higher Education Ministry for providing a $\mathrm{PhD}$ grant to Mr. Nabil Sabor at Niigata University, Electrical and Electronic Engineering Department.

\section{References}

[1] J. Yick, B. Mukherjee, and D. Ghosal, "Wireless Sensor Network Survey," Computer Networks, vol. 52, pp. 2292-2330, 2008.

[2] Y.-M. Huang, M.-Y. Hsieh, and F. Sandnes, "Wireless Sensor Networks and Applications," in Sensors. vol. 21, S. C. Mukhopadhyay and R. Y. M. Huang, Eds., ed: Springer Berlin Heidelberg, 2008, pp. 199219.

[3] Y. Liu, "Wireless Sensor Network Applications in Smart Grid: Recent Trends and Challenges," International Journal of Distributed Sensor Networks, vol. 2012, pp. 1-8, 2012.

[4] B. Rashid and M. H. Rehmani, "Applications of Wireless Sensor Networks for Urban Areas: A Survey," Journal of Network and Computer Applications, vol. 60, pp. 192-219, 2016.

[5] M. P. Đurišić, Z. Tafa, G. Dimić, and V. Milutinović, "A survey of Military Applications of Wireless Sensor Networks," in 2012 Mediterranean Conference on Embedded Computing (MECO), Bar, 2012, pp. 196-199.

[6] J. Ko, C. Lu, M. B. Srivastava, J. A. Stankovic, A. Terzis, and M. Welsh, "Wireless Sensor Networks for Healthcare," Proceedings of the IEEE, vol. 98, pp. 1947-1960, 2010.

[7] J. Valverde, V. Rosello, G. Mujica, J. Portilla, A. Uriarte, and T. Riesgo, "Wireless Sensor Network for Environmental Monitoring: Application in a Coffee Factory," International Journal of Distributed Sensor Networks, vol. 2012, pp. 1-18, 2012.

[8] G. Xu, W. Shen, and X. Wang, "Applications of Wireless Sensor Networks in Marine Environment Monitoring: A Survey," Sensors (Basel), vol. 14, pp. 16932-16954, 2014.

[9] A. Flammini, P. Ferrari, D. Marioli, E. Sisinni, and A. Taroni, "Wired and Wireless Sensor Networks for Industrial Applications," Microelectronics Journal, vol. 40, pp. 1322-1336, 2009.

[10] F. Fabio, C. A. C. Coello; and M. Repetto, "Multiobjective Optimization and Artificial Immune System: A Review," in Handbook of research on Artificial Immune Systems and Natural Computing: Applying Complex Adaptive Technologies, ed: IGI Publishing, 2009, pp. 1-21.

[11] H. Lu, "An Adaptive Multi-objective Immune Optimization Algorithm," in Control, Automation and Systems Engineering, 2009. CASE 2009. IITA International Conference on, Zhangjiajie, 2009, pp. 140143.

[12] W. R. Heinzelman, A. Chandrakasan, and H. Balakrishnan, "Energy-Efficient Communication Protocol for Wireless Microsensor Networks," in System Sciences, 2000. Proceedings of the 33rd Annual Hawaii International Conference on, 2000, pp. 1-10.

[13] J.-L. Liu and C. V. Ravishankar, "LEACH-GA: Genetic Algorithm-Based Energy-Efficient Adaptive Clustering Protocol for Wireless Sensor Networks," International Journal of Machine Learning and Computing, vol. 1, pp. 79-85, 2011.

[14] K. G. Vijayvargiya and V. Shrivastava, "An Amend Implementation on LEACH protocol based on Energy Hierarchy," International Journal of Current Engineering and Technology, vol. 2, pp. 427-431, 2012.

[15] M. Abo-Zahhad, S. M. Ahmed, N. Sabor, and S. Sasaki, "A New Energy-Efficient Adaptive Clustering Protocol Based on Genetic Algorithm for Improving the Lifetime and the Stable Period of Wireless Sensor Networks," International Journal of Energy, Information and Communications, vol. 5, pp. 47-72, 2014. 
[16] B. Baranidharan and B. Santhi, "GAECH: Genetic Algorithm Based Energy Efficient Clustering Hierarchy in Wireless Sensor Networks," Journal of Sensors, vol. 2015, p. 8, 2015.

[17] Y. K. Tamandani and M. U. Bokhari, "SEPFL Routing Protocol Based on Fuzzy Logic Control to Extend the Lifetime and Throughput of the Wireless Sensor Network," Wirel. Netw., vol. 22, pp. 647653, 2016.

[18] G. Smaragdakis, I. Matta, and A. Bestavros, "SEP: A Stable Election Protocol for Clustered Heterogeneous Wireless Sensor Networks," in Second International Workshop on Sensor and Actor Network Protocols and Applications (SANPA 2004), 2004, pp. 1-11.

[19] Y. Yao and N. Jiang, "Distributed Wireless Sensor Network Localization Based on Weighted Search," Computer Networks, vol. 86, pp. 57-75, 2015

[20] G. Wu, S. Wang, B. Wang, Y. Dong, and S. Yan, "A Novel Range-Free Localization Based on Regulated Neighborhood Distance for Wireless Ad Hoc and Sensor Networks," Computer Networks, vol. 56, pp. 3581-3593, 2012.

[21] M. N. Halgamuge, M. Zukerman, K. Ramamohanarao, and H. L. Vu, "An Estimation of Sensor Energy Consumption," Progress In Electromagnetics Research B, vol. 12, pp. 259-295, 2009.

[22] R. K. a. Jyotishree, "Blending Roulette Wheel Selection \& Rank Selection in Genetic Algorithms," Machine Learning and Computing, vol. 2, pp. 365-370, 2012.

[23] M. Abo-Zahhad, S. M. Ahmed, N. Sabor, and A. F. Al-Ajlouni, "The Convergence Speed of Single- and Multi-Objective Immune Algorithm Based Optimization Problems," Signal Processing : An International Journal, vol. 4, pp. 247-303, 2010.

[24] W. Z. Khan, N. M. Saad, and M. Y. Aalsalem, "An Overview of Evaluation Metrics for Routing Protocols in Wireless Sensor Networks," in Intelligent and Advanced Systems (ICIAS), 2012 4th International Conference on, Kuala Lumpur 2012, pp. 588-593.

[25] L. Alazzawi and A. Elkateeb, "Performance Evaluation of the WSN Routing Protocols Scalability," Journal of Computer Systems, Networks, and Communications, vol. 2008, 2008.

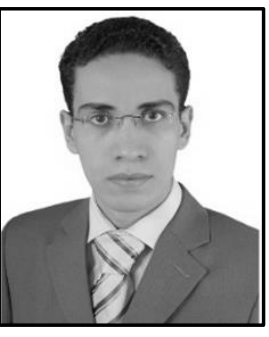

\section{Authors}

Nabil Sabor, he received the B.S.E.E. (Hons.) and M.S.E.E. degrees in electrical engineering from Assiut University, Egypt, in 2006 and 2011, respectively. Since 2006, he has been with Assiut University, where he is currently an Assistant Lecturer with the Department of Electronics and Communication Engineering. He is also a Ph.D. Researcher with the Department of Electrical and Electronic Engineering, Niigata University, Japan. He has authored over 15 papers in national and international journals and conferences in his research fields. His research interests include signal and image processing, data compression, biomedical signal processing, wavelet transform, digital filters, genetic algorithms, immune algorithms, and wireless sensor networks.

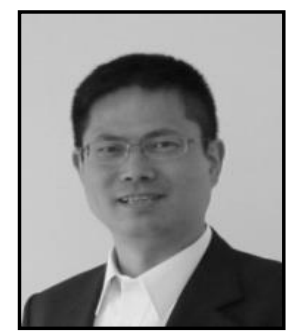

Shigenobu Sasaki, he received B.E., M.E. and Ph.D. degrees from Nagaoka University of Technology, Nagaoka, Japan, in 1987, 1989 and 1993, respectively. Since 1992, he has been with Niigata University, where he is now a Professor at the Department of Electrical and Electronic Engineering. From 1999 to 2000, he was a visiting scholar at the Department of Electrical and Computer Engineering, University of California, San Diego. From 2003 to 2006, he was with the UWB technology institute, National Institute of Information and Communication Technology (NICT) as an expert researcher, where he was involved the R\&D on UWB wireless 


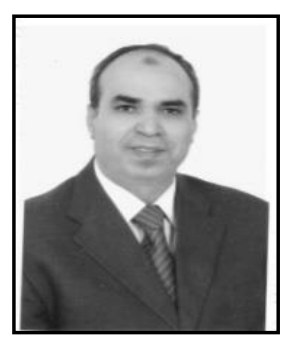

communications and standardization activities in ITU-R TG1/8. His research interests are in the area of wideband digital communications including spread spectrum systems, ultrawideband wireless communications and cognitive radio technology. $\mathrm{He}$ is also actively involved in the IEEE 802 standardization on wireless communications over white space, such as the IEEE $802.15 .4 \mathrm{~m}$ and the IEEE 802.22 . He is a member of IEEE.

Mohammed Abo-Zahhad, he (SM'00) received the B.S.E.E. and M.S.E.E. degrees in electrical engineering from Assiut University, Egypt, in 1979 and 1983, respectively, and the Ph.D. degree from the University of Kent, Canterbury, U.K., and Assiut University (channel system), in 1988. He was the Vice Dean of Graduated Studies from 2006 to 2012. He has been a Professor of Electronics and Communication Engineering since 1999. He has been the Head of the Electrical Engineering Department since 2013. He has authored over 130 papers in national and international journals and conferences in his research fields. His research interests include switchedcapacitor, optical and digital filters biomedical and genomic signal processing, speech processing, data compression, wavelet-transforms, genetic algorithms, immune algorithms, wireless sensor networks, and electronic systems. $\mathrm{He}$ is a member of the European Society of Circuit Theory and Applications in 1998.

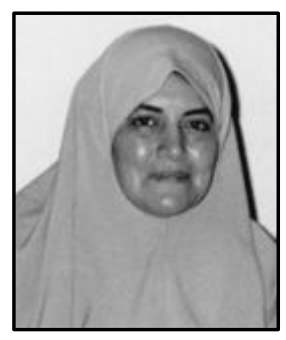

Sabah M. Ahmed, she received the B.S.E.E. (Hons.) and M.S.E.E. degrees in electrical engineering from Assiut University, Egypt, in 1979 and 1983, respectively, and the Ph.D. degree from the Technical University of Budapest, Hungary, in 1992. She has been a Professor of Electronics and Communication Engineering since 2009, and the Manager with the Communication and Information Technology Training Center, Assiut University, since 2010. She has authored over 75 papers in national and international journals and conferences in her research fields. Her research interests include speech processing, biomedical and genomic signal processing, data compression, wavelet-transforms, genetic algorithms, and immune algorithms 